\title{
Intermittent theta burst stimulation facilitates functional connectivity from the dorsal premotor cortex to primary motor cortex
}

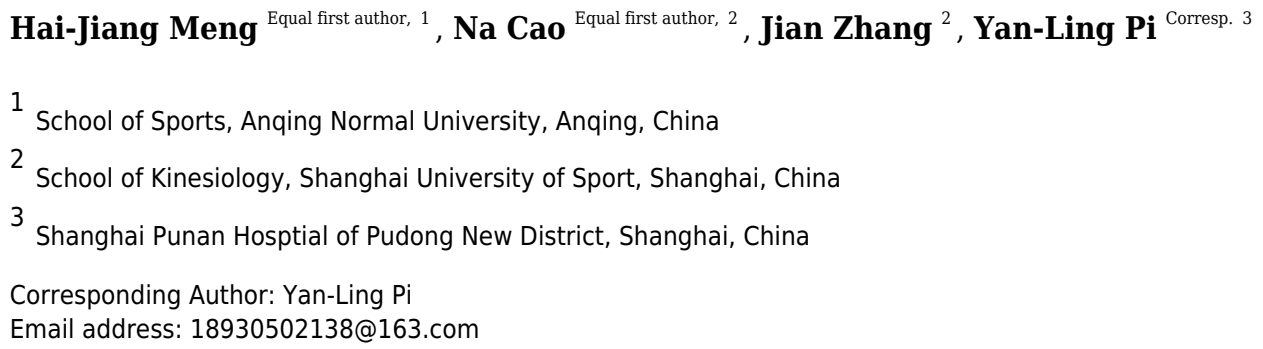

Background. Motor information in the brain is transmitted from the dorsal premotor cortex (PMd) to the primary motor cortex (M1), where it is further processed and relayed to the spinal cord to eventually generate muscle movement. However, how information from the PMd affects M1 processing and the final output are unclear. Here, we applied intermittent theta burst stimulation (iTBS) to the PMd to alter cortical excitability not only at the application site but also at the PMd projection site of M1. We aimed to determine how PMd iTBS-altered information changed M1 processing and the corticospinal output.

Methods. In total, 16 young, healthy participants underwent PMd iTBS with 600 pulses (iTBS600) or sham-iTBS600. Corticospinal excitability, short-interval intracortical inhibition (SICI), and intracortical facilitation (ICF) were measured using transcranial magnetic stimulation before and up to 60 min after stimulation.

Results. Corticospinal excitability in M1 was significantly greater 15 min after PMd iTBS600 than that after sham-iTBS600 ( $p=0.012)$. Compared with that after sham-iTBS600, at $0(p=0.014)$ and $15(p=$ 0.037 ) min after iTBS600, SICI in M1 was significantly decreased, whereas 15 min after iTBS600, ICF in M1 was significantly increased $(p=0.033)$.

Conclusion. Our results suggested that projections from the PMd to M1 facilitated M1 corticospinal output and that this facilitation may be attributable in part to decreased intracortical inhibition and increased intracortical facilitation in M1. Such a facilitatory network may inform future understanding of the allocation of resources to achieve optimal motion output. 
1 Intermittent theta burst stimulation facilitates functional connectivity from

2 the dorsal premotor cortex to primary motor cortex

3

4

5 Hai-Jiang Meng ${ }^{1}, \mathrm{Na} \mathrm{Cao}^{2}$, Jian Zhang ${ }^{2}$, Yan-Ling $\mathrm{Pi}^{3}$

6

7

8

${ }^{1}$ School of Sports, Anqing Normal University, Anqing, China

9

${ }^{2}$ School of Kinesiology, Shanghai University of Sport, Shanghai, China

${ }^{3}$ Shanghai Punan Hosptial of Pudong New District, Shanghai, China

Corresponding author:

Yan-Ling Pi

Shanghai Punan Hospital, Pudong New District, Shanghai, China.

Email address: 18930502138@163.com 
After a period of noninvasive brain stimulation or certain patterns of activity, the function as well

as the very structure of the cortex of the brain changes. These functional and structural cortical changes occur through a process known as cortical plasticity (Pascual-Leone et al., 2005). Fundamental processes, such as motor learning, depend on neuronal plasticity occurring in a number of brain regions, especially among those areas that are spatially interconnected (Luber \& Lisanby, 2014). However, recruitment of the functional connectivity that makes up the crucial interneuron networks among these interconnected regions differs (Nettekoven et al., 2014).

A growing body of research has shown that a broad set of key brain regions, known as the motor system network, are functionally involved in motor performance and motor control. This motor system network is not restricted to the motor cortex but dynamically extends into parietal, temporal, and prefrontal areas, depending on task complexity and an individual's experience (Swinnen \& Wenderoth, 2004). The major functional area of the motor system, the dorsal premotor cortex (PMd), is crucial for controlling the preparation and execution of motor behaviors (Roth et al., 1996). The corticocortical connections from the PMd to the ipsilateral primary motor cortex (M1) are thought to transmit information relevant to generate the final motor output (Koch et al., 2007). However, how the PMd-M1 neural circuits achieve this output remains poorly understood, particularly after dynamic modifiability by external perturbation.

Methodological advances in noninvasive brain stimulation techniques - such as transcranial magnetic stimulation (TMS) and repetitive TMS (rTMS) - have informed our knowledge of cortical plasticity and its underlying mechanisms (Ni et al., 2011). Single-pulse TMS activates interneurons, and this activation discharges corticospinal neurons, generating motor-evoked potentials (MEPs) in the target muscle that can be used to assess the level of corticospinal 
82

83

84

excitability (Hallett, 2007). A different type of TMS protocol, called paired-pulse TMS, can be used to probe intracortical circuits that are highly interconnected and to determine the final motor cortical output. Short-interval intracortical inhibition (SICI), a well-investigated intracortical inhibitory phenomenon, can be elicited when a subthreshold conditioning stimulation suppresses a subsequent suprathreshold test stimulation (at interstimulus intervals of 1-5 ms), leading to inhibition of the subsequent MEP. Intracortical facilitation (ICF) is elicited with a similar protocol but using longer interstimulus intervals of 6-30 ms (Kujirai et al., 1993). Studies have reported that the balance and interaction between intracortical inhibition and facilitation are critical for the regulation of neuronal excitability and plasticity (Dai et al., 2016). Intermittent theta burst stimulation (iTBS), a type of rTMS, alters motor cortex excitability. This altered excitability has been shown by an increase in the amplitude of MEPs measured at the innervated target muscle for as long as 20 min following single-pulse TMS (Cardenas-Morales et al., 2010; Huang et al., 2005). In addition, rTMS is capable of evoking aftereffects at distant sites (such as M1) that are interconnected with the stimulated cortex (such as PMd). These remote aftereffects have been attributed to effective activation of output and input connections between the PMd and M1 (Bestmann et al., 2003; Rizzo et al., 2004). Thus, the results of such studies demonstrate that PMd and M1 interconnections can be assessed by TMS measurement of cortical excitatory changes in M1.

The application of rTMS not only influences the characteristics of neurons within the stimulated region but also may affect activity levels of remote but interconnected areas, and this remote change is a long-lasting aftereffect (Bestmann et al., 2003). For example, a study using TMS combined with magnetic resonance imaging showed that TMS with anterior-posterior directed current applied to M1 strengthens the connectivity between the premotor and M1 regions (Volz et al., 2015). These stimulation-induced changes in M1 are attributable to both local factors and the interconnections with the premotor region (Cardenas-Morales et al., 2014). In addition, a previous study using TMS combined with electroencephalography showed that cerebellar TBS 
109

110

111

112

113

114

115

116

117

118

119

120

121

122

123

124

125

126

127

128

129

130

131

132

133

134

135

modulated cortical excitability of distant interconnected motor areas through common temporal, spatial and frequency domains (Casula et al., 2016). These findings suggest that a relationship exists between the responsiveness to rTMS and the motor network connectivity (Koch et al., 2007).

In the present study, we investigated whether the aftereffects induced by iTBS on the PMd affected the excitability of the ipsilateral M1 and its intracortical circuits. We hypothesized that the aftereffects induced by a specific iTBS protocol would increase the excitability of M1 and would decrease the degree of the intracortical inhibition. We further hypothesized the existence of a facilitatory network from the stimulated PMd to the ipsilateral M1.

\section{Materials and Methods}

\section{Participants}

We studied 16 right-handed, healthy participants ( 8 women and 8 men; mean age, $22.19 \pm 1.72$ years) with no history of neurological or psychiatric diseases. Right-handedness was determined based on the Edinburgh Handedness Inventory (Oldfield, 1971). All participants provided written informed consent in accordance with the Declaration of Helsinki. The protocol was approved by the ethics committee of the Shanghai University of Sport (reference No. SUS2014024).

\section{Electromyographic recording}

To record electromyogram signals from a selected target muscle, the first dorsal interosseous (FDI) muscle of the right hand, $\mathrm{Ag}-\mathrm{AgCl}$ surface electrodes (9 $\mathrm{mm}$ in diameter) were placed at the tendon belly. The signal was bandpass filtered $(20 \mathrm{~Hz}$ to $2.5 \mathrm{kHz})$ and then amplified 1000 times (Intronix Technologies amplifier, model 2024F). The signal was then digitized at $5 \mathrm{kHz}$ using a Micro1401 data acquisition unit (Cambridge Electronics Design, Cambridge, UK). The resulting data were analyzed with Signal software, version 6.02 . 
136

137

138

139

140

141

142

143

144

145

146

147

148

149

150

151

152

153

154

155

156

157

158

159

160

161

162

\section{Transcranial magnetic stimulation}

We generated a monophasic current that traveled in a posterior to anterior direction in the left hemisphere M1 by using coils (9.5 $\mathrm{cm}$ in diameter) with a figure-eight shape that were connect to a stimulator (Magstim 2002; Whitland, Dyfed, UK). The coil handle was held so that is was approximately $90^{\circ}$ to the central sulcus and at a $45^{\circ}$ angle to the central sagittal line. The coil was moved until a suprathreshold stimulation of the left hemisphere M1 generated the highest MEP amplitude in the target muscle, the right FDI. The position of the coil was then marked.

The measurements recorded included the resting motor threshold (RMT), MEP amplitude (a measure of the degree of corticospinal excitability), SICI, and ICF that were induced with a paired-pulse TMS paradigm (Ni et al., 2011). We defined RMT as the minimum stimulation necessary to induce MEPs at the FDI that had amplitudes measured from peak to peak of at least $50 \mu \mathrm{V}$ in 5 or more of 10 trials while the FDI muscle was relaxed. To evaluate iTBS-induced MEP amplitude changes, the amplitude was compared with that before iTBS using a 1-mV TMS intensity. This $1-\mathrm{mV}$ intensity was defined as the lowest TMS intensity needed to generate MEPs with amplitudes greater than $1 \mathrm{mV}$ in 5 or more of 10 trials while the FDI muscle was relaxed. For the SICI and ICF paired-pulse TMS paradigms, they were consisted of a subthreshold condition pulse set as 70\%RMT and a suprathreshold test pulse set at $1 \mathrm{mV}$ intensity and were adjusted as needed at each time point to maintain the amplitude of test MEPs at approximately $1 \mathrm{mV}$, but left the conditioning intensity unchanged (Huang et al., 2005; Ni et al., 2014). The interstimulus interval was $2 \mathrm{~ms}$ for SICI and $10 \mathrm{~ms}$ for ICF. The interstimulus interval of $2 \mathrm{~ms}$ for SICI was selected because initial testing of intervals from 1 to $5 \mathrm{~ms}$ indicated that the MEP amplitude showed peak suppression at this time (Kujirai et al., 1993). Similarly, the interstimulus interval of $10 \mathrm{~ms}$ for ICF was selected because $10 \mathrm{~ms}$ is the most effective interval to induce ICF (Ni et al., 2011). Ten trials for each measurement (i.e., MEP amplitude, SICI and ICF) were conducted in a random order. 
163

164

165

166

167

168

169

170

171

172

173

174

175

176

177

178

179

180

181

182

183

184

185

186

187

188

189

\section{Theta burst stimulation}

The present study followed a previously described iTBS protocol with a slight modification (Huang et al., 2005). Briefly, one burst was defined as three pulses given at a frequency of $50 \mathrm{~Hz}$. We gave 10 bursts every $200 \mathrm{~ms}$ for $2 \mathrm{~s}$. Ten more bursts were given every $10 \mathrm{~s}$ for $191 \mathrm{~s}$ to generate a total of 600 pulses; thus, this protocol is termed iTBS600 herein. To prevent preactivation of the FDI muscle, we decreased the intensity of the iTBS used in the original protocol (which was $80 \%$ of the individual's active motor threshold) to $70 \%$ of the RMT (Gentner et al., 2008; Huang et al., 2008). Previous studies using this modification have reported aftereffects consistent with studies using the original stimulation (Gentner et al., 2008;

Cárdenas-Morales et al., 2014).

In the present study, TBS stimulation was delivered to the left hemisphere PMd. The PMd was defined as the area $2.5 \mathrm{~cm}$ anterior and $1 \mathrm{~cm}$ medial to the left hemisphere $\mathrm{M} 1_{\text {Hand }}$ area measured from the scalp (Mochizuki et al., 2010).

\section{Experimental design}

To investigate how cortical excitability and plasticity reorganization affect the motor system network after iTBS is applied to the PMd, we conducted two experimental sessions for each participant: iTBS600 applied to the PMd, and sham-iTBS600 applied to the PMd. The shamiTBS600 protocol consisted of sham stimulation blocks. To reduce potential cortical stimulation effects for these sham sessions, the coil was held at $45^{\circ}$, with the rim opposite the handle of the coil — rather than the center of the coil — touching the skull. Previous studies have reported that this is an effective sham stimulation method because the coil-cortex distance is relatively large, substantially reducing the electromagnetic field (Cao et al., 2018).

The order of the sessions in which either PMd iTBS600 or PMd sham-iTBS600 was applied was counterbalanced among the participants. Participants were asked to wait at least 2 weeks between 
190

191

192

193

194

195

196

197

198

199

200

201

202

203

204

205

206

207

208

209

210

211

212

213

214

215

216

the first and the second experimental sessions to avoid potential confounding of the results. The experimental design is shown in Fig. 1. Measurements were taken before (baseline) as well as 0 , 15, 30, and $60 \mathrm{~min}(\mathrm{~T} 0, \mathrm{~T} 15, \mathrm{~T} 30, \mathrm{~T} 60)$ after each interventional protocol.

\section{Data and statistical analyses}

To examine the effects of iTBS600 or sham-iTBS600 and time on the MEP amplitude and on SICI or ICF, we used two-way repeated-measures analyses of variance (ANOVAs). Protocols (iTBS vs sham-iTBS) and time (baseline, T0, T15, T30, T60) were within-subject factors included in the ANOVAs. Significant findings were further probed with post hoc Bonferroni tests, which corrected for multiple comparisons.

All statistical analyses were conducted using SPSS software, version 17.0, and two-sided values of $p<0.05$ indicated statistically significant differences. We reported values herein as the mean \pm standard error.

\section{Results}

The measurements of MEP amplitude, SICI, ICF did not show significant differences (all $p>$ 0.05) for the different interventional protocols at baseline.

\section{Corticospinal M1 excitability}

We examined whether the level of corticospinal M1 excitability (the final motor output) was altered when iTBS600 was applied to the PMd by measuring the change in MEP amplitude at the target FDI muscle after the application. A two-way repeated-measures ANOVA revealed a significant main effect of time $\left(F_{(4,60)}=6.767, p=0.002\right)$ as well as a significant interaction between interventional protocol and time $\left(F_{(4,60)}=3.431, p=0.014\right)$; however, the main effect of interventional protocol was not significant $\left(F_{(1,15)}=1.72, p=0.209\right)$. Post hoc tests indicated that compared with the application of sham-iTBS600 to the PMd, application of iTBS600 
217 significantly increased MEP amplitude $15 \mathrm{~min}$ after the intervention $(p=0.012)$. Meanwhile, 218 compared with the baseline, application of iTBS600 to the PMd significantly increased MEP 219 amplitude in M1 15 min after the intervention $(p=0.004)$. These results suggested that 220 corticospinal M1 excitability was significantly increased by iTBS600 applied to the PMd (Fig. 2).

\section{Intracortical circuits}

223 We next examined whether application of iTBS600 to the PMd altered either the inhibitory or 224 facilitatory intracortical M1 circuits by measuring SICI and ICF, respectively, as determined by

225

226

227

228

229

230

231

232

233

234

235

236

237

238

239

240

241

243

242 Discussion the MEP amplitude change in the target FDI muscle evoked by paired-pulse stimulation. For SICI, A two-way repeated-measures ANOVA found that both the main effect of time $\left(F_{(4,60)}=\right.$ $3.247, p=0.018)$ and the interaction between time and interventional protocol $\left(F_{(4,60)}=5.257, p\right.$ $=0.001)$ were significant, whereas the main effect of interventional protocol was not $\left(F_{(1,15)}=\right.$ $3.016, p=0.103)$. Post hoc tests indicated that compare with the application of sham-iTBS600 to the PMd, application of iTBS600 to the PMd significantly decreased SICI in M1 at 0 min ( $p=$ $0.014)$ and at $15 \min (p=0.037)$ after the application, suggesting that iTBS600 applied to the PMd decreased inhibitory intracortical MI circuits (Fig. 3).

For assessment of ICF, a two-way repeated-measures ANOVA found that neither the main effect of time $\left(F_{(4,60)}=1.298, p=0.281\right)$ nor of interventional protocol $\left(F_{(1,15)}=0.906, p=0.356\right)$ was significant. However, the interaction between time and interventional protocol was significant $\left(F_{(4,60)}=3.087, p=0.022\right)$. Post hoc tests indicated that compared with sham-iTBS600 applied to the PMd, iTBS600 applied to the PMd significantly increased ICF in M1 at $15 \min (p=0.033)$ after the application, suggesting that iTBS600 applied to the PMd increased activity at facilitatory intracortical MI circuits (Fig. 4).

In the present study, we used rTMS to examine whether the excitability of intracortical circuits in 
244 M1 were changed by the aftereffects of iTBS600 applied to the PMd. Our primary findings were 245 that compared with sham-iTBS600 applied to the PMd, 15 min after iTBS600 was applied to the

246 PMd (to induce alterations in cortical excitability beyond a relatively short stimulation period),

247 corticospinal M1 excitability measured via the change in MEP amplitude was significantly

248 higher, and the degree of the facilitation among the intracortical circuits measured via a change

249 in ICF was significantly increased. In addition, immediately as well as 15 min after this iTBS600

250 application, the degree of the inhibition among the intracortical circuits (intrinsic to the motor

251 cortex) measured via a change in SICI was significantly decreased.

252

253

Neurophysiology of premotor-motor connections

254 Synaptic activity between interconnected brain regions is altered even at rTMS intensity levels lower than the cortical threshold intensity (Bestmann et al., 2003). A previous study has shown that iTBS applied to M1 increases MEP amplitude at the target muscle for approximately 15 min (Huang et al., 2005). Diverse stimulation protocols may involve long-term potentiation (LTP)like and long-term depression (LTD)-like processes not only in the motor cortex but also in nonmotor cortex in vivo (Chung et al., 2017). Previous studies have highlighted the role of Nmethyl-D-aspartate (NMDA) receptors as key mediators of excitability changes and excitatory synaptic transmission in the brain, which could explain the aftereffects of TBS on neuronal circuitry (Huang et al., 2005). Application of this model to our results would suggest that iTBS modulation of the PMd enhanced its excitability and induced an LTP-like effect as well as an increase in MEP amplitude and a decrease in SICI in M1. The increase in MEP amplitude and decrease in SICI in M1 suggested that the effectiveness of the synaptic connections was increased by iTBS applied to the PMd, which induced an LTP-like effect. These changes are consistent with increased activation of NMDA receptors in the PMd and decreased gammaaminobutyric acid (GABA)ergic levels in M1 and suggest that LTP-like effects in the PMd may be associated with LTD-like effects in M1. At the synaptic level, the fine balance between excitation (mediated by glutamate) and inhibition (mediated by GABA) is crucial for optimal 
271 neuroplasticity (Dai et al., 2016). In the present study, increased MEP amplitude was observed

$27215 \mathrm{~min}$ after the iTBS protocol was applied, whereas SICI decreased immediately after iTBS.

273 The speeds at which the various receptors involved activate their effectors and the amounts of

274 the neurotransmitters released may have contributed to these results. Excitatory interneurons

275 receive inputs from inhibitory interneurons that mediate SICI (Ni et al., 2011), with many

276 connections organized in a center-surround pattern to initiate point to point facilitation and

277 widespread inhibition. Such an arrangement would suggest more possibilities for transmission

278 from inhibitory rather than facilitatory receptors, leading to an earlier plasticity change

279 (Hanajima et al., 2001).

280

281 iTBS aftereffects on intracortical circuits

282 During TMS, the MEP amplitude at the muscle is determined by M1 output. This output is a 283 complex product of interactions between excitatory facilitation and inhibitory actions of the 284 stimulated region as well as networks within M1 itself and other motor-related cortical areas 285 connected with M1. In the present study, the decrease in SICI and increase in ICF observed after 286 iTBS of the PMd suggested that the effectiveness of the synaptic connections increased in M1. 287 Previous work has shown that enhanced $\mathrm{GABA}_{\mathrm{A}}$ receptor activation increases SICI (Ziemann et 288 al., 1996). Given this finding, the decreased SICI observed in the present study could reasonably

289 be attributed to activation of $\mathrm{GABA}_{\mathrm{A}}$ receptors. The final output relies on the interplay between 290 the inhibitory inputs and excitatory inputs projected onto the corticospinal neurons. Thus, the

291

292

293

294

295

296

297 observed increased M1 activity may be associated with an iTBS-induced increase in postsynaptic activity, similar to that related to the LTP that is also mediated by $\mathrm{GABA}_{\mathrm{A}}$ receptors. However, TBS at intervals of $200 \mathrm{~ms}$ ( $5 \mathrm{~Hz}$ theta rhythm) has been shown to promote LTP by decreasing inhibitory input, enabling larger NMDA receptor responses both presynaptically and postsynaptically (Davies et al., 1991; Thickbroom, 2007). Such a trans-synaptic modulation of the iTBS-stimulated PMd would greatly contribute to the later effects we observed (at $15 \mathrm{~min}$ ) at the PMd projection site of M1. Thus, a TBS-induced presence of GABAergic activity at the 
298 intracortical level may cause a change in the excitability of another brain region without any

299 stimulation of that latter region.

\section{Enhancement of motor abilities through noninvasive stimulation}

301 The PMd was selected for this study because it is important for the precise timing and execution

302 of unimanual and coordinated bimanual movements (Ni et al., 2009). Although the present study

303 focused solely on the physiological data, our data offer an undergirding mechanism for future

304 work involving behavioral-level analyses to advance understanding of motor learning and

305 control.

306

307 Previous research has indicated that 50-Hz triple-pulse rTMS of the PMd increases both motor

308 function and motor learning; for example, the ability to learn and to perform a motor sequence task or a skilled grasping task is increased (Gregori et al., 2005). TMS applied over the left PMd has been shown in one study to decrease the lack of grip strength as well as to inhibit corticospinal excitability. The authors of that study suggested that the effect of blocking the PMd preparatory activity indicated a causal role for the PMd in grip force (Duque et al., 2010). Future investigations will be needed to clarify how TBS is associated with motor learning at the behavior level.

315

Our study was not without limitations that may affect the interpretation of our results. A main limitation was the absence of a neuronavigation system to accurately localize the target areas during the experimental sessions. A second limitation was that we used a relatively small number of participants who were close in age, restricting the generalizability of the results to younger or older individuals. Therefore, future research should aim for a larger number of participants and different age groups.

\section{Conclusions}

324 The present study provided evidence in support of our hypothesis that the application of iTBS to 
the PMd increases excitability and decreases SICI in M1. These results suggested that a facilitatory network exists from the PMd to the M1. The existence of such a network informs the understanding of the allocation of resources to achieve optimal motion output.

\section{References}

Bestmann S, Baudewig J, Siebner HR, Rothwell JC, and Frahm J. 2003. Subthreshold high-frequency TMS of human primary motor cortex modulates interconnected frontal motor areas as detected by interleaved fMRI-TMS. Neuroimage 20:1685-1696 DOI 10.1016/j.neuroimage.2003.07.028.

Cao N, Pi Y, Liu K, Meng H, Wang Y, Zhang J, Wu Y, and Tan X. 2018. Inhibitory and facilitatory connections from dorsolateral prefrontal to primary motor cortex in healthy humans at rest-An rTMS study. Neuroscience Letters 687:82-87 DOI 10.1016/j.neulet.2018.09.032.

Cardenas-Morales L, Nowak DA, Kammer T, Wolf RC, and Schonfeldt-Lecuona C. 2010. Mechanisms and applications of theta-burst rTMS on the human motor cortex. Brain Topogr 22:294-306 DOI 10.1007/s10548-009-0084-7.

Cardenas-Morales L, Volz L, Michely J, Rehme AK, Pool E-M, Nettekoven C, Eickhoff SB, Fink GR, and Grefkes C. 2014. Network Connectivity and Individual Responses to Brain Stimulation in the Human Motor System. Cerebral Cortex 24:1697-1707 DOI 10.1093/cercor/bht023.

Casula EP, Pellicciari MC, Ponzo V, Bassi MS, Veniero D, Caltagirone C, and Koch G. 2016. Cerebellar theta burst stimulation modulates the neural activity of interconnected parietal and motor areas. Scientific reports 6 : 36191.

DOI 10.1038/srep36191

Chung SW, Lewis BP, Rogasch NC, Saeki T, Thomson RH, Hoy KE, Bailey NW, Fitzgerald PB. 2017. Demonstration of short-term plasticity in the dorsolateral prefrontal cortex with theta burst stimulation: a TMS-EEG study. Clinical Neurophysiology 128:1117-1126

Dai W, Pi YL, Ni Z, Tan XY, Zhang J, Wu Y. 2016. Maintenance of balance between motor cortical excitation and inhibition after long-term training. Neuroscience 336:114-122 DOI 10.1016/j.neuroscience.2016.08.053.

Davies CH, Starkey SJ, Pozza MF, and Collingridge GL. 1991. GABA autoreceptors regulate the induction of LTP. Nature 349:609-611 DOI 10.1038/349609a0.

Duque J, Lew D, Mazzocchio R, Olivier E, and Ivry RB. 2010. Evidence for two concurrent inhibitory mechanisms during response preparation. J Neurosci 30:3793-3802 DOI 10.1523/JNEUROSCI.5722-09.2010.

Gentner R, Wankerl K, Reinsberger C, Zeller D, and Classen J. 2008. Depression of Human Corticospinal Excitability Induced by Magnetic Theta-burst Stimulation: Evidence of Rapid Polarity-Reversing Metaplasticity. Cerebral Cortex 18:2046-2053 
DOI 10.1093/cercor/bhm239.

Gregori B, Currà A, Dinapoli L, Bologna M, Accornero N, and Berardelli A. 2005. The timing and intensity of transcranial magnetic stimulation, and the scalp site stimulated, as variables influencing motor sequence performance in healthy subjects. Experimental Brain Research 166(1):43-55 DOI 10.1007/s00221-005-2337-3.

Hallett M. 2007. Transcranial magnetic stimulation: a primer. Neuron 55:187-199 DOI 10.1016/j.neuron.2007.06.026

Hanajima R, Ugawa Y, Machii K, Mochizuki H, Terao Y, Enomoto H, Furubayashi T, Shiio Y, Uesugi H, Kanazawa I. 2001. Interhemispheric facilitation of the hand motor area in humans. J Physio/ 531:849-859 DOI 10.1111/j.1469-7793.2001.0849h.x

Huang YZ, Edwards MJ, Rounis E, Bhatia KP, and Rothwell JC. 2005. Theta burst stimulation of the human motor cortex. Neuron 45:201-206 DOI 10.1016/j.neuron.2004.12.033.

Huang YZ, Rothwell JC, Edwards MJ, and Chen RS. 2008. Effect of physiological activity on an NMDA-dependent form of cortical plasticity in human. Cereb Cortex 18:563-570 DOI 10.1093/cercor/bhm087.

Koch G, Franca M, Mochizuki H, Marconi B, Caltagirone C, and Rothwell JC. 2007. Interactions between pairs of transcranial magnetic stimuli over the human left dorsal premotor cortex differ from those seen in primary motor cortex. Journal of Physiology (Oxford) 578(2):551-562

DOI 10.1113/jphysiol.2006.123562

Kujirai T, Caramia MD, Rothwell JC, Day BL, Thompson PD, Ferbert A, Wroe S, Asselman P, and Marsden CD. 1993. Corticocortical inhibition in human motor cortex. Journal of Physiology 471:501-519 DOI 10.1113/jphysiol.1993.sp019912.

Luber B, and Lisanby SH. 2014. Enhancement of human cognitive performance using transcranial magnetic stimulation (TMS). Neuroimage 85(3):961-970 DOI 10.1016/j.neuroimage.2013.06.007.

Mochizuki H, Huang YZ, and Rothwell JC. 2010. Interhemispheric interaction between human dorsal premotor and contralateral primary motor cortex. Journal of Physiology 561:331-338 DOI 10.1113/jphysiol.2004.072843.

Nettekoven C, Volz L, Kutscha M, Pool EM, Rehme AK, Eickhoff SB, Fink GR, and Grefkes C. 2014. Dosedependent effects of theta burst rTMS on cortical excitability and resting-state connectivity of the human motor system. J Neurosci 34:6849-6859 DOI 10.1523/JNEUROSCI.4993-13.2014.

Ni Z, Gunraj C, Nelson AJ, Yeh IJ, Castillo G, Hoque T, and Chen R. 2009. Two phases of interhemispheric inhibition between motor related cortical areas and the primary motor cortex in human. Cereb Cortex 19:1654-1665 DOI 10.1093/cercor/bhn201.

Ni Z, Müller-Dahlhaus F, Chen R, and Ziemann U. 2011. Triple-pulse TMS to study interactions between neural circuits in human cortex. Brain Stimulation 4:281-293 DOI 10.1016/j.brs.2011.01.002.

Ni Z, Gunraj C, Kailey P, Cash RF, and Chen R. 2014. Heterosynaptic modulation of motor cortical plasticity in human. Journal of Neuroscience 34(21): 7314-7321. 
405

406

407

408

409

410

411

412

413

414

415

416

417

418

419

420

421

422

423

424

425

426

427

428

429

430

DOI 10.1523/JNEUROSCI.4714-13.2014

Oldfield RC. 1971. The assessment and analysis of handedness: the Edinburgh inventory. Neuropsychologia 9:97113 DOI 10.1016/0028-3932(71)90067-4.

Pascual-Leone A, Amedi A, Fregni F, and Merabet LB. 2005. The plastic human brain cortex. Ann Rev Neurosci 28:377-401

DOI 10.1146/annurev.neuro.27.070203.144216.

Rizzo V, Siebner HR, Modugno N, Pesenti A, Munchau A, Gerschlager W, Webb RM, Rothwell JC. 2004. Shaping the excitability of human motor cortex with premotor rTMS. J Physiol 554:483-495 DOI 10.1113/jphysiol.2003.048777

Roth M, Decety J, Raybaudi M, Massarelli R, Delon-Martin C, Segebarth C, Morand S, Gemignani A, Décorps M, and Jeannerod M. 1996. Possible involvement of primary motor cortex in mentally simulated movement: a functional magnetic resonance imaging study. Neuroreport 7:1280-1284 DOI 10.1097/00001756-199605170-00012.

Swinnen SP, and Wenderoth N. 2004. Two hands, one brain: cognitive neuroscience of bimanual skill. Trends in Cognitive Sciences 8:18-25 DOI 10.1016/j.tics.2003.10.017.

Thickbroom GW. 2007. Transcranial magnetic stimulation and synaptic plasticity: experimental framework and human models. Experimental Brain Research 180:583-593 DOI 10.1007/s00221-007-0991-3

Volz L, Hamada M, Rothwell JC, and Grefkes C. 2015. What Makes the Muscle Twitch: Motor System Connectivity and TMS-Induced Activity. Cereb Cortex 25:2346-2353 DOI 10.1093/cercor/bhu032.

Ziemann U, Lönnecker S, Steinhoff BJ, and Paulus W. 1996. The effect of lorazepam on the motor cortical excitability in man. Experimental Brain Research 109:127-135 DOI 10.1007/bf00228633 


\section{Figure 1}

Figure 1. Schematic of the experimental design.

The four measurements, resting motor threshold (RMT), motor-evoked potential (MEP) amplitude, intracortical facilitation (ICF), and short-interval intracortical inhibition (SICI), were obtained before (at baseline) as well as immediately and up to $60 \mathrm{~min}$ after application of the interventional protocols. iTBS indicates intermittent theta burst stimulation, and PMd represents dorsal premotor cortex.

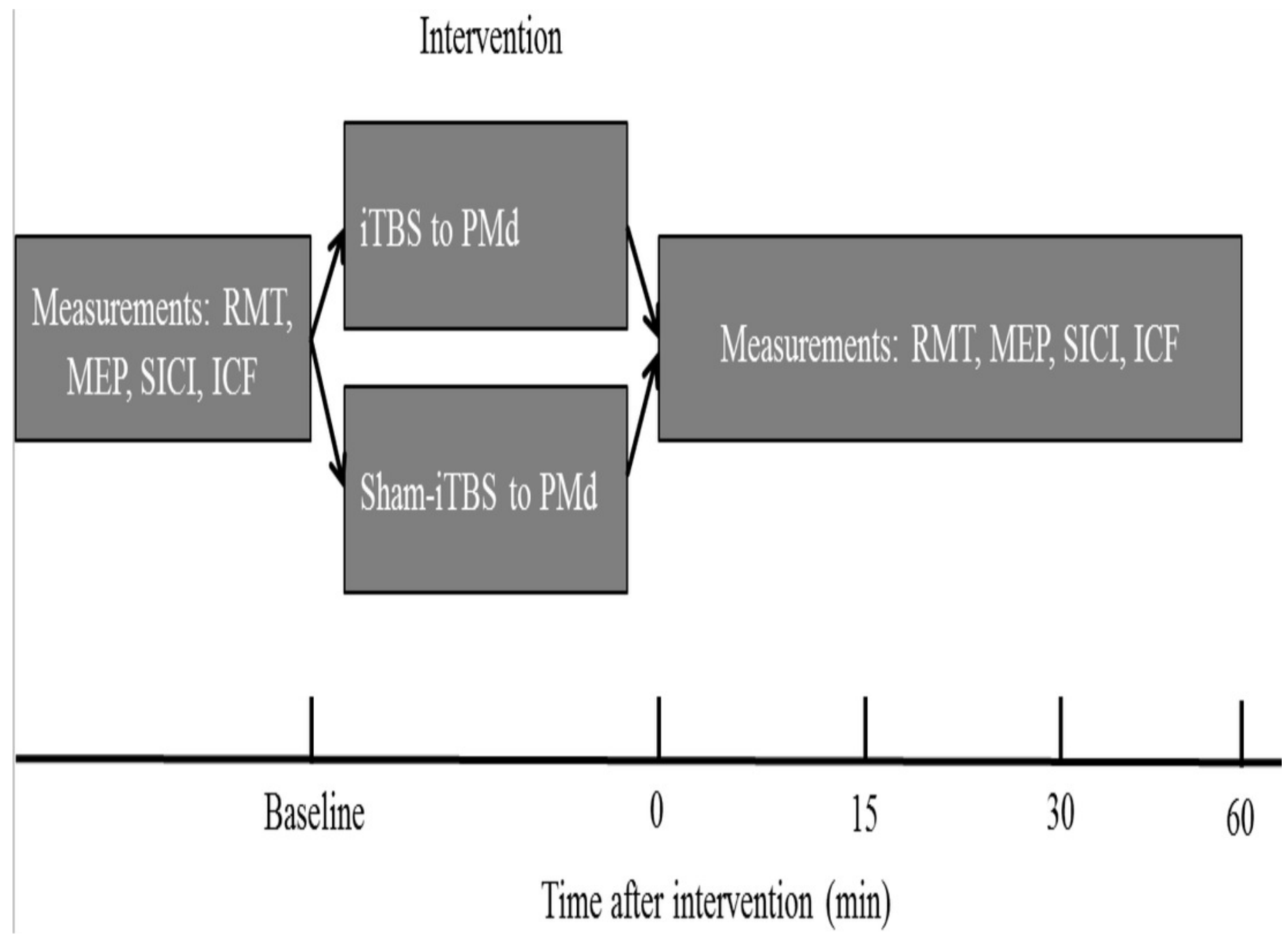




\section{Figure 2}

Figure 2. Effect of intermittent theta burst stimulation (iTBS)-induced dorsal premotor cortex (PMd) on primary motor cortex (M1) excitability.

MEP amplitudes were measured peak to peak. Circles indicate means and standard errors of the MEP amplitudes before and after sham-iTBS600 applied to the PMd interventional protocol. Black triangles indicate means and standard errors of the MEP amplitudes before and after iTBS600 applied to the PMd interventional protocol. ${ }^{*} p<0.05$ compared with shamiTBS. 


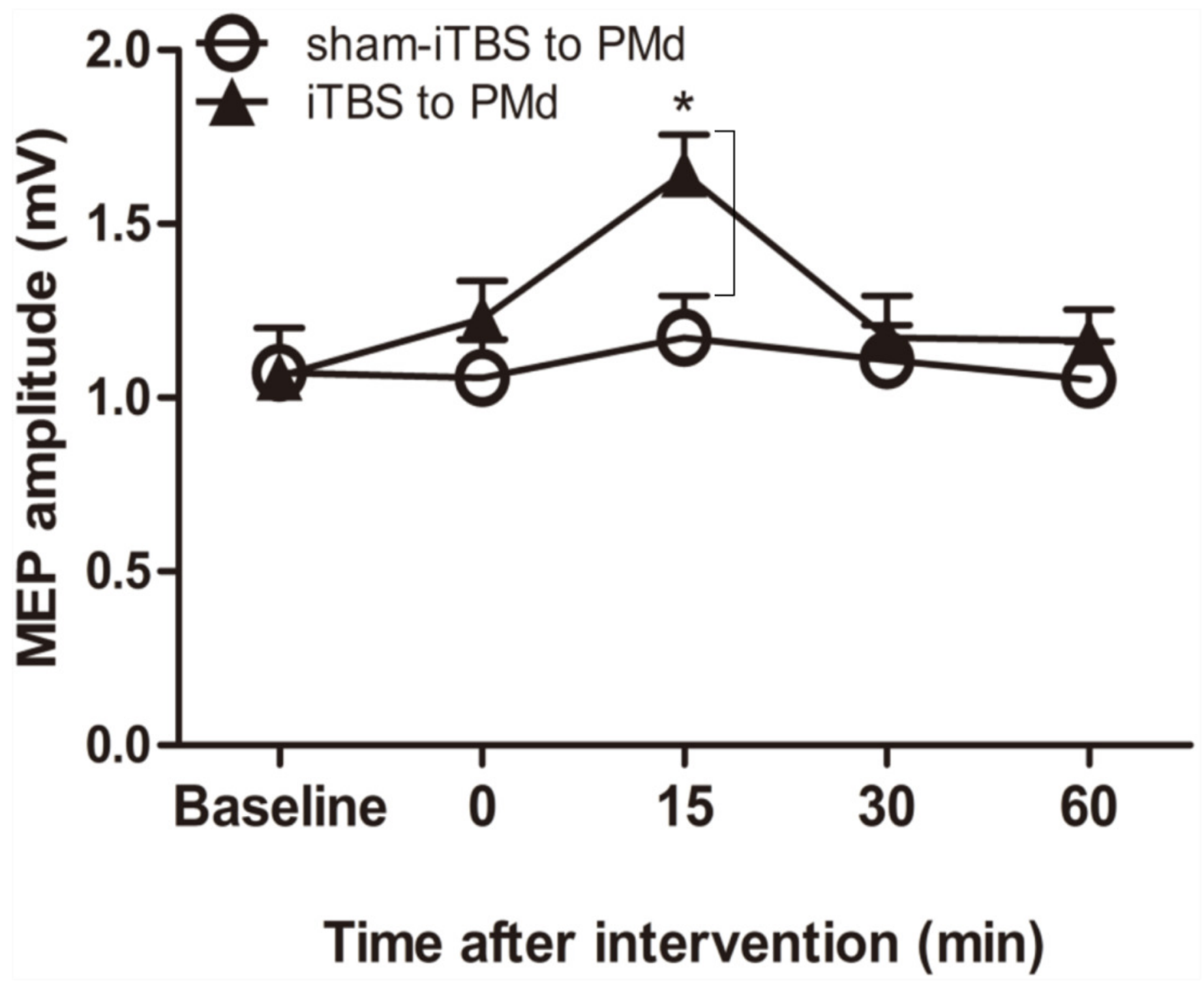




\section{Figure 3}

Figure 3. Effects of intermittent theta burst stimulation (iTBS) applied to the dorsal premotor cortex (PMd) on the inhibitory intracortical circuits of the primary motor cortex.

The effect on short-interval intracortical inhibition ( $\mathrm{SICl}$ )was investigated by determining the conditioned motor-evoked potential (MEP) amplitudes expressed as the percentage of the MEP amplitudes induced by the test stimulus at baseline alone. Circles indicate means and standard errors of the SICl before and after sham-iTBS600 applied to the PMd interventional protocol. Black triangles indicate means and standard errors of the $\mathrm{SICl}$ before and after iTBS600 applied to the PMd interventional protocol. * $p<0.05$ compared with sham-iTBS. 


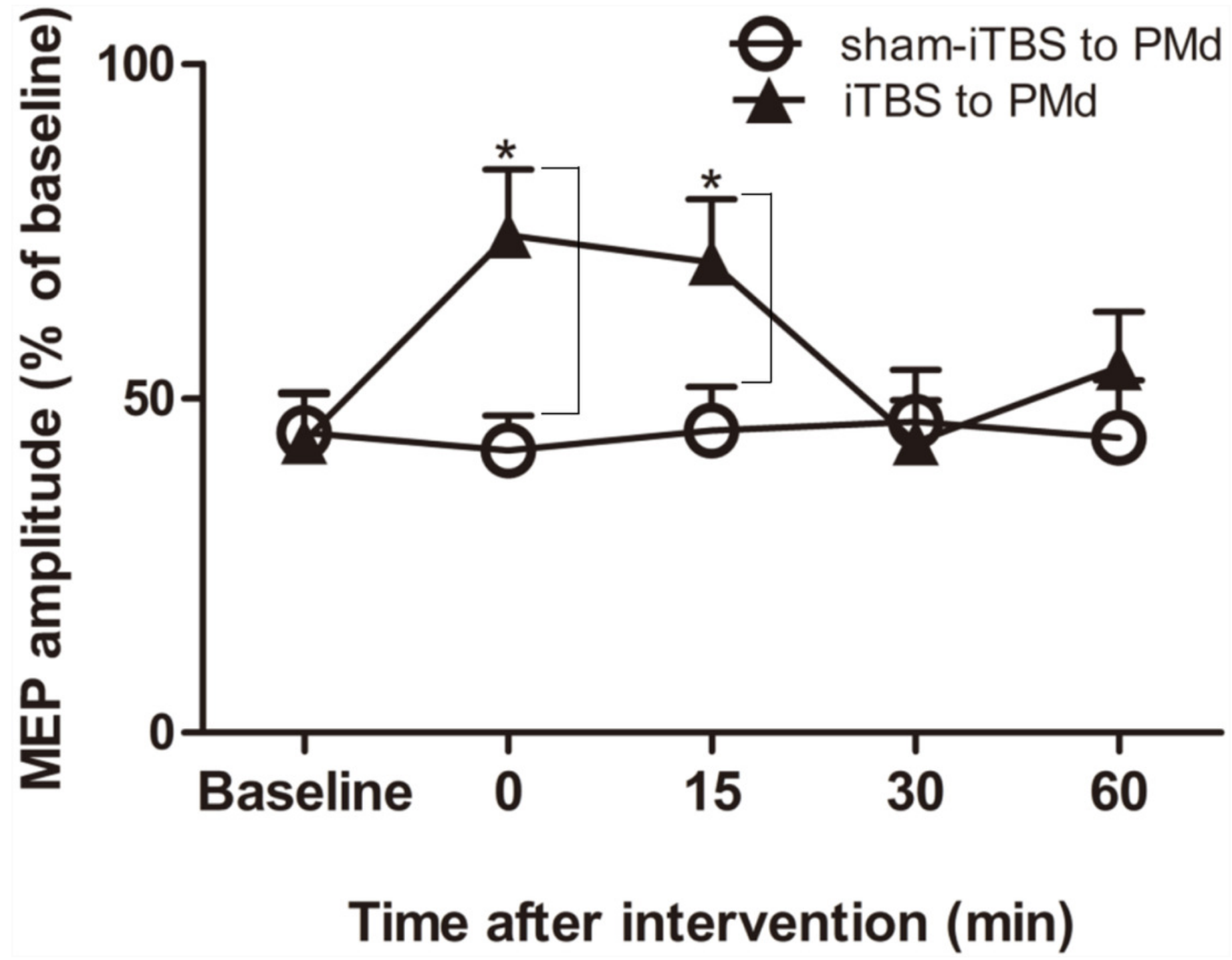




\section{Figure 4}

Figure 4. Effects of intermittent theta burst stimulation (iTBS) applied to the dorsal premotor cortex (PMd) on faciliatory intracortical circuits of the primary motor cortex.

The effect on intracortical facilitation (ICF) was investigated by determining the conditioned motor-evoked potential (MEP) amplitudes expressed as a percentage of the MEP amplitudes induced by the test stimulus at baseline alone. Circles indicate means and standard errors of the ICF before and after application of the sham-iTBS600 interventional protocol to the PMd. Black triangles indicate means and standard errors of the ICF before and after the iTBS600 interventional protocol was applied to the PMd. * $p<0.05$ compared with sham-iTBS600. 


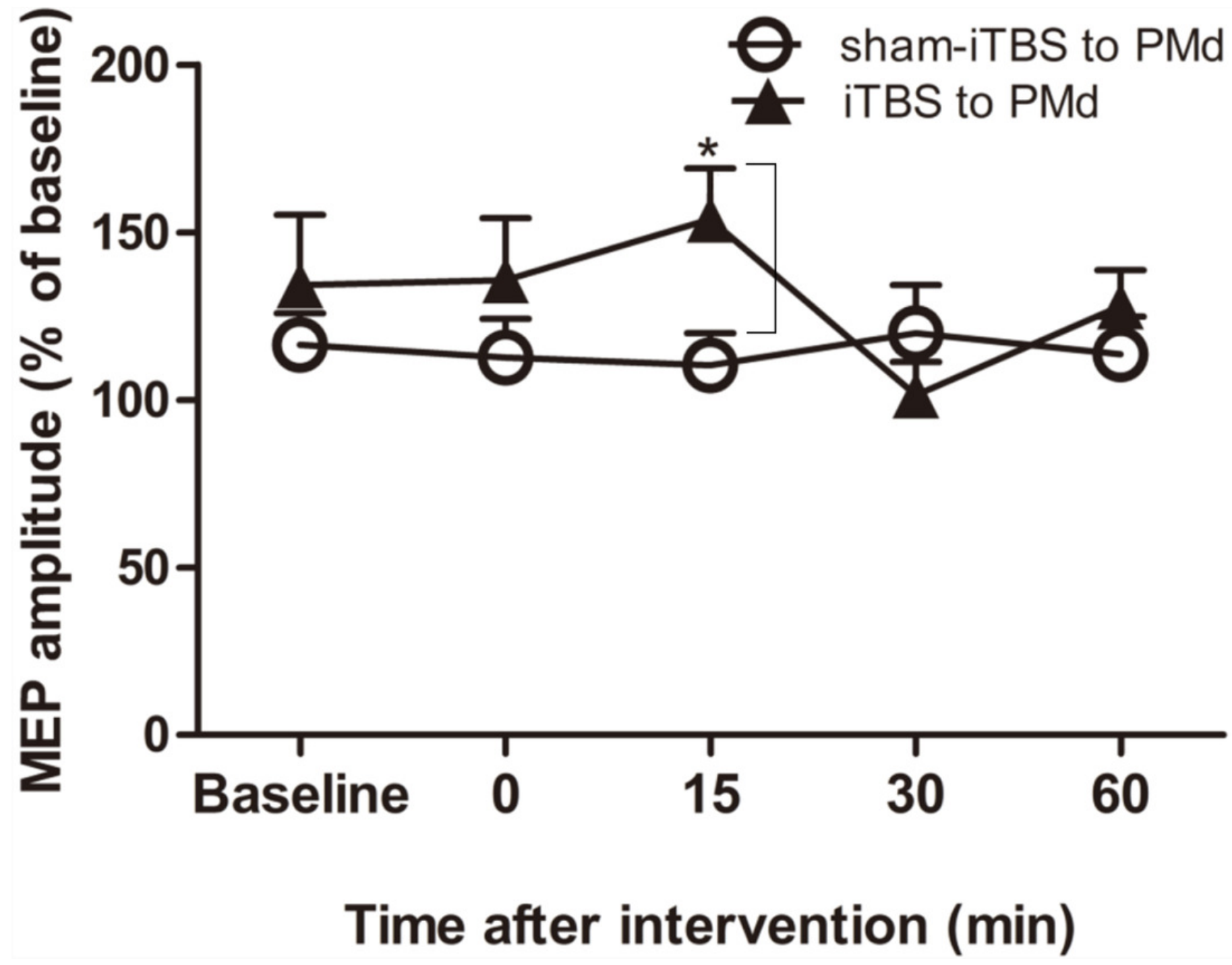

